\title{
Percepciones sobre el tránsito a modalidades de enseñanza no presenciales. Un estudio exploratorio entre docentes de educación pública básica en la ciudad de Guadalajara, México Perceptions About Passing Through to a New Teaching Modality. An Exploratory Study Among Public Secondary School Teachers in Guadalajara, Mexico
}

Es doctoranda en Educación por la Universidad de Guadalajara con una investigación en torno a los significados de docentes y directivos sobre el fenómeno de inclusión y exclusión educativa; maestra en Estudios Psicoanalíticos Freudianos en Espacio Psicoanalítico; y licenciada en Psicología por la UNIVA.

\section{RODRIGO GONZÁLEZ REYES}

Es doctor en Comunicación por la Universidad Nacional de La Plata, Argentina; maestro en Comunicación por la Universidad de Guadalajara; y licenciado en Ciencias de la Comunicación por el ITESO. Es investigador del Departamento de Estudios de la Comunicación Social de la Universidad de Guadalajara, e investigador titular en la Cátedra UNESCO AMIDI. Es también editor adjunto de la revista Comunicación y Sociedad, y secretario de Documentación de la Asociación Mexicana de Investigadores de la Comunicación (AMIC). 



\section{Percepciones sobre el tránsito a modalidades de enseñanza no presenciales. Un estudio exploratorio entre docentes de educación pública básica en la ciudad de Guadalajara, México Perceptions About Passing Through to a New Teaching Modality. An Exploratory Study Among Public Secondary School Teachers in Guadalajara, Mexico}

Talía Chávez Palencia y Rodrigo González Reyes

Universidad de Guadalajara, México

taliach@gmail.com (https://orcid.org/0000-0003-2870-5080)

rodrigo@suv.udg.mx (https://orcid.org/0000-0003-0142-9522)

Recibido: 04-02-2021 / Aceptado: 11-11-2021

https://doi.org/10.18800/conexion.202102.003

PALABRAS CLAVE / KEYWORDS

Modalidades no presenciales, percepciones, docentes, educación pública, educación básica, COVID-19 / non presential modalities, perceptions, teachers, public education, basic education, COVID-19

\section{RESUMEN}

Este estudio exploratorio tiene como finalidad identificar las principales percepciones de docentes de educación pública básica en la ciudad de Guadalajara, México, en torno al proceso de migración a modalidades no presenciales durante el inicio de la pandemia por la COVID-19. La meta general tras el diseño del trabajo empírico fue crear un corte sincrónico sobre la presencia de estas percepciones para poder comparar, en estudios posteriores y a mediano plazo, las posibles oscilaciones en las percepciones entre los mismos docentes y, probablemente, entre otro tipo de actores.

\section{ABSTRACT}

The aim of this exploratory study, ran in Guadalajara city, Mexico, is to identify, among public education teachers, about the migration process to non presential teaching modalities. The methodological goal was to provide a solid and instantaneous cut in the middle of the experience of these teachers to get the opportunity to compare, in the long term, the evolution of those perceptions. 


\section{Percepciones sobre el tránsito a modalidades de enseñanza no presenciales. Un estudio exploratorio entre docentes de educación pública básica en la ciudad de Guadalajara, México}

Desde el 25 de octubre de 2005, se celebra mundialmente el Día de Internet. De esta reciente efemérides se han ido desdoblando y derivando iniciativas públicas que pugnan por la inclusión digital, el derecho al acceso a la información, y el fortalecimiento de las ciudadanías digitales y la educación integral en la digitalidad; con todo, los números no son alentadores: la expansión de internet y sus extensiones y el equipamiento social y tecnológico no sobrepasan por mucho las dotaciones de hace una década en la inmensa mayoría de países subdesarrollados, mientras que los indicadores de desarrollo no muestran una salud digna de presumirse (Medina Mayagoitia et al., 2017). Como se sabe, este hecho ha impactado con fuerza en los sectores profesionalizados de la educación y ha evidenciado lo que ya era más que visible: la tecnologización no ha llegado a la educación y los problemas estudiados en el campo de la comunicación son mucho más amplios y complejos de lo que generalmente se incorpora a las agendas académicas.

Este punto se vuelve especialmente crítico para lo que aquí se trabaja, pues, ante la pandemia por la COVID-19, que surgió sin
2020, la tabla de flotación de las prácticas educativas a la que saltamos y nos aferramos desde entonces y hasta estos días han sido las «plataformas digitales», tanto las que en su origen están pensadas con fines educativos como aquellas que no.

Así, desde los cruces de la micromensajería de WhatsApp y Messenger con los medios de videoconferencia como Zoom y Google Meet hasta la implementación protagónica de las plataformas modulares -Classroom, Microsoft Teams, Moodle, Canva y una decena más-, muchos docentes arribaron al mundo de la plataformización de la educación por primera vez - y demasiadas de las veces - sin referencias prácticas.

Decimos que este punto se vuelve crítico pues, si bien las ideas de plataforma y plataformización se encuentran hoy en día en el catálogo de las cosas posibles - hace una década seguramente que no-, el problema se desarrolla no en torno a su disponibilidad, sino a las condiciones de acceso y aprovechamiento práctico de estas (Srnicek, 2019).

Mientras que la plataformización educativa, entendida en palabras de Schenone como «un proceso, en el cual el funcionamiento "normal" de la escuela tal cual la conocemos se va hibridizando con la incorporación de plataformas, tanto de aprendizaje como sociales, en las interacciones de la enseñanza y el aprendizaje» (2020, p. 3), avanza de manera implacable 
como una opción económica y transversal en todos los ámbitos educativos, particularmente en el institucional, este proceso se ha caracterizado por resultar abiertamente desigual y parcelador: ha encontrado a grandes segmentos del magisterio desprovistos de equipamiento tecnológico, de los más básicos contextos y referencias técnicas y de las más esenciales habilidades digitales.

Desde ahí, podemos ver, no solo la precarización del acceso a internet ha ido generando una visible y peligrosa brecha entre aquellos que son capaces de acceder a ella y los que no, sino también en torno a aquellos que poseen o no dominio y destreza técnica sobre el entorno de plataformas, aptitudes que no están garantizadas por el hecho de formar parte del segmento de quienes acceden a internet (Medina Mayagoitia et al., 2017).

Desde aquí, y entendiendo que la plataformización de la educación implica un entorno particular que reclama habilidades, tecnicidades y destrezas especiales (Schenone, 2020), hemos querido enmarcar un problema educativo que se liga naturalmente a todo lo anterior: las percepciones sobre las dificultades y los problemas -así como las posibles bondades- de un apresurado movimiento migratorio del magisterio de enseñanza pública hacia las modalidades virtuales o en línea, basadas en estas plataformas, y el tratamiento prácti- co de las tecnologías educativas en medio de esta contingencia sanitaria.

En este sentido, y como nota metodológica, es importante señalar que, si bien este estudio tiene como propósito central el posar la mirada en un momento y corte cronológico particular - el año 2020, en el que se inició la pandemia-, la literatura existente sobre el desarrollo de los fenómenos educativos en este contexto, en torno a experiencias empíricas más transversales, es amplia; por ejemplo, puede revisarse aquella contenida en las antologías dedicadas a este problema en el número 5, volumen 22, de la Revista Digital Universitaria de la Universidad Nacional Autónoma México ${ }^{1}$, o el monográfico publicado recientemente por la Revista Argentina de Investigación Educativa de la Universidad Nacional de La Plata en su volumen 1 , número $1^{2}$, entre muchas otras e importantes iniciativas documentales y empíricas -incluida la edición del presente número de Conexión- que pueden ser consultadas por país o región e, incluso, por periodos a lo largo del desarrollo de la pandemia.

\section{Marco teórico}

\section{Vitrina metodológica}

Uno de los problemas metodológicos centrales en el estudio de los fenómenos sociales históricos es la llamada distorsión retrospectiva, ese efecto a partir del cual lo

${ }^{1}$ Los artículos del número están disponibles en https:// www.revista.unam.mx/2021v22n5/.

${ }^{2}$ La publicación se encuentra en https://portalrevistas.unipe.edu.ar/index.php/raie/issue/view/1/2. 
sucedido en el pasado es reconstruido de manera poco clara debido a la pérdida de elementos descriptivos que le eran sustanciales (Gallagher, 2018); otro de estos problemas es la obliteración por asimilación. Este es un mecanismo de enmarcamiento en el que los hechos trascendidos, luego de haber sido revisados y reactualizados a través de tantas narrativas, pierden parte de su esencia a partir de la reinvención de versiones (Watts, 2012).

Aunque los efectos de estos sesgos son imposibles de parar, pues se trata de mecanismos sustanciales a la cognición humana, diferentes estrategias de investigación e intervención metodológica resultan muy útiles en la tarea de permitir una interpretación a posteriori del hecho y ayudar a fijar una posición de lo sucedido (Morgan y Winship, 2007/2014); estas estrategias son maniobras de documentación in vivo, rutas metodológicas que dan lugar a investigaciones intencionalmente diseñadas para lograr capturar un corte en el momento en que un hecho social se mantiene en desarrollo para, posteriormente, poder compararlo e imbricarlo en los hechos consecutivos.

Justamente, este trabajo ha tenido esa intención metodológica: intentar atrapar y retener la perspectiva de los docentes de escuelas de educación básica pública, en medio de un momento clave en el desarrollo de un acontecimiento crítico: la migración intempestiva y no planeada a modalidades de enseñanza no presencia- les durante el surgimiento y avance de la pandemia por la COVID-19.

Con profesores rebasados en sus labores y responsabilidades, con estudiantes en situaciones de recepción educativa precarizada, con padres de familia o tutores intentando salvar lo básico frente a lo urgente y con infraestructuras tecnoeducativas desmanteladas $u$ obsolescentes, el panorama general, desde este lado de la realidad, no puede verse más que negro; no obstante, una mirada menos discreta revela, como sucedió en este estudio, la existencia de dinámicos procesos de negociación de estos actores frente a la adversidad y diferentes percepciones que están lejos, incluso, del gris en el panorama. Poder retener estos cortes «tomográficos» nos permitirá -y esa es la apuesta- rescatar esas negociaciones y percepciones e ir divisando las oscilaciones y centros, al mediano y largo plazo, sobre esas apreciaciones y sentires.

\section{Intencionalidad del estudio}

La intención tras este trabajo exploratorio, que se llevó a cabo durante siete semanas entre junio y julio de 2020 -lapso que incluyó la aplicación de entrevistas, y su codificación y sistematización-, no ha sido explicar una experiencia cerrada y superada por parte de sus protagonistas, sino, por el contrario, generar una fotografía instantánea a partir de un corte sincrónico al inicio de la emergencia sanitaria por la COVID-19 en torno a las percepciones de 
algunos docentes de educación pública básica que lograron migrar totalmente a modalidades no presenciales, y específicamente acerca de lo más relevante para ellos durante el proceso de transición mientras las condiciones aún están frescas en sus prácticas y primeras apreciaciones.

Así, se partió del supuesto de que esta investigación, de tipo aquí y ahora, podrá aportar, a mediano y largo plazos, un referente empírico inicial sobre la configuración de un fenómeno en plena evolución; y que, dadas las condiciones, podrá servir para observar su posterior desarrollo y ayudar a reconstruir un mapa más amplio y diverso a partir de distintas visiones asociadas a las prácticas educativas durante esta crisis.

\section{Metodología}

\section{Diseño de investigación}

Considerando que esta situación ha irrumpido de manera inesperada y que, hasta el momento en que esto se escribe -finales de octubre de 2021-, la pandemia continúa vigente, la pregunta que guio el trabajo de campo fue la siguiente: ¿cuáles han sido, para los docentes en estudio, las situaciones percibidas como más importantes durante el proceso de tránsito de la modalidad presencial a las no presenciales?

Tras esta pregunta está el interés por conocer no solo las situaciones, sino también qué eventos o circunstancias quedan relacionadas con ellas como docentes de educación pública básica. Desde ahí, el estudio se planeó y diseñó como exploratorio, y tuvo como meta identificar los principales núcleos temáticos con relación a los aspectos vivenciales que los docentes, desde la posición de haber podido migrar en su totalidad, reconocen como trascendentes, bien porque fueron problemáticos o por lo contrario (Gauntlett, 2007, pp. 16-22).

Con la intención de que la exploración se desarrollara de la manera más descriptiva posible y poniendo al centro a los propios docentes para que fueran informantes y sujetos empíricos a la vez, se optó por un diseño inductivo que facilitara la producción de categorías nativas y el surgimiento de ejes temáticos a priori desde las propias narrativas, en vez de tomar un marco teórico y sus categorías preestablecidas (Hammersley, 1992).

\section{Selección del caso y proceso de segmentación}

Al principio del planeamiento del diseño, se abrieron dos grandes opciones: trabajar con docentes de distintas escuelas públicas en una muestra ampliada, o únicamente con docentes pertenecientes a una misma escuela y construir un caso único de estudio; aunque la primera opción resultaba muy tentadora por la amplitud de las variaciones descriptivas que podía ofrecer en tanto que aumentaba la diversidad de perfiles y contextos de los docen- 
tes, esta elección presentaba el gran inconveniente, para el tiempo que se tenía, de requerir extender la muestra a niveles de saturación descriptiva y la posterior necesidad de categorizar los relatos por segmentos.

Se dice que el tiempo era corto porque, como se señaló, el objetivo era generar una fotografía instantánea mientras la situación se mantuviera activa y fresca en las prácticas y percepciones de los docentes, y dado que las vacaciones de verano y el cambio de ciclo escolar amenazaban la continuidad de esta etapa; el tiempo de trabajo de campo y sistematización quedó sujeto a un periodo entre la publicación oficial en Jalisco del decreto que suspendía las clases por la pandemia - el 13 de marzo- y las vacaciones de Semana Santa - Pascua -del 6 al 18 de abril-, en el límite inferior; y el inicio de las vacaciones de verano - segunda semana de julio, con variaciones según cada institución-, en el superior. Como se puede ver, el tiempo era el principal apremio: 10 semanas para todo el proceso de pesquisa.

Ante estas circunstancias, se optó por la segunda opción; en este caso, el criterio de saturación descriptiva se sustituyó por el de relevancia heurística (Saldaña, 2009/2015, p. 8), para lo cual la primera condición necesaria fue la adhesión voluntaria al estudio por parte de los docentes - por lo tanto, se trató de un muestreo por conveniencia- (Auerbach y Silverstein, 2003, p. 18).
Una vez tomada esta decisión, se buscaron planteles regulares de la Zona Metropolitana de la ciudad de Guadalajara -Jalisco, México-, la cual es la capital y ciudad más poblada del estado y la segunda más grande del país después de la capital (Instituto Nacional de Estadística y Geografía, 2018, p. 19), y donde residimos nosotros los investigadores.

Tras varios intentos en diferentes escuelas, pudimos tener acceso a una de las que cuenta con mayor cantidad de alumnos inscritos en esa zona escolar. Dado que la escuela se encontraba físicamente cerrada por la contingencia, el contacto con los profesores se llevó a cabo a través de un reclutamiento de bola de nieve y con la asesoría de una directiva del plantel en el proceso de identificación. El criterio principal para poder integrarse a la muestra fue una combinación de dos factores: (1) haber podido migrar totalmente de la modalidad presencial a las no presenciales; $y$ (2) integrarse voluntariamente al estudio, como ya se ha comentado. El número final de profesores que pudieron migrar de manera total fue de seis; todos ellos accedieron a colaborar como voluntarios a través de entrevistas no presenciales.

Respecto a la escuela, esta cuenta con 24 grupos de primero a tercero de secundaria, con un promedio de 40 alumnos por salón en dos turnos: matutino y vespertino. En promedio, trabajan 100 docentes, la mayor parte en tiempo variable. De esta población, tan solo seis profesores -tres 
hombres y tres mujeres- lograron hacer la transición total a plataformas y modalidades no presenciales.

\section{Diseño metodológico}

Parte central del diseño fue segmentar la muestra a partir de elegir a profesores que lograron migrar de manera exitosa. Se trató de un muestreo por conveniencia porque no se trabajó con todos los profesores del plantel, sino solo con aquellos que voluntariamente se adhirieron el estudio (Auerbach y Silverstein, 2003, p. 8), y porque contempló solo a aquellos que se inscribieron plenamente en la condición de haber migrado totalmente.

Como técnica de levantamiento de datos, se eligió la entrevista en etapas, ya que se buscaba, principalmente, que afloren percepciones valoradas antes que profundizar en esas percepciones (Edwards y Holland, 2013, p. 32); a la vez, se pretendía lograr entrevistas que permitieran la producción de núcleos temáticos comunes y un final cerrado en tiempos relativamente cortos (Seidman, 2006, p. 16), sobre todo tomando en cuenta que estas entrevistas, debido a la cuarentena, se llevarían a cabo a través de una plataforma de videoconferencia en línea. Con estas condiciones, la ruta metodológica comprendió dos fases:

(1) mapeo de conceptos categoriales y (2) heurística del fenómeno.

En la primera etapa, se sondeó la presencia de temas salientes entre los infor- mantes, con lo que se confeccionó la primera colección de categorías del estudio -ejes-. Esta primera etapa se basó en producir concepciones nativas a través de códigos in vivo a partir de propiciar la imposición de dominio - entendida esta como la capacidad de los informantes y sujetos empíricos de nombrar los fenómenos investigados con etiquetas y categorías propias y generadas nativamente- de manera individual, con lo que se planteó un mapa general de conceptos.

En la segunda fase, al tratarse de entrevistas en etapas, la intervención se enfocó en identificar las percepciones predominantes sobre los significados individuales concedidos a los conceptos centrales para, luego, pasar a que les atribuyeran un sentido propio que se anclara en su experiencia cotidiana. Las entrevistas resultantes se codificaron de manera general abierta según el modelo estándar de Strauss y Corbin (Auerbach y Silverstein, 2003), que contempla la identificación de categorías salientes a partir de buscar códigos representativos para el fenómeno trabajado (Saldaña, 2009/2015, p. 20).

\section{Contexto del caso de estudio}

La Secretaría de Educación Pública federal, en México, anunciaba la cuarentena para los diferentes sistemas educativos del país a partir del 23 de marzo de 2020 . Esta se pensaba mantener hasta el 17 de abril (Acuerdo Número 02/03/20, 2020), situación que no sucedió, pues el confi- 
namiento se ha alargado hasta el momento en que se escribe este estudio. Ese día, también se puso en marcha el programa Aprende en casa, estrategia que, en reemplazo de las clases presenciales, incorporaba modalidades de enseñanza a distancia vía radio, televisión e internet («“Aprende en casa TV”», 2020).

Para el 21 de abril, esta dependencia oficial ofreció un seminario web inaugural titulado Presentación de la Nueva Escuela Mexicana en línea: desaprendiendo para aprender, el cual fue presidido por el secretario de Educación Pública, Esteban Moctezuma Barragán, y en el que se anunció la alianza recién establecida entre esta secretaría, Google for Education y la plataforma de contenidos YouTube con el fin de hacer más asequibles estas tecnologías de cara a un proceso de capacitación docente.

Por su parte, y en un pasado cercano -en el año 2019-, el Gobierno estatal de Jalisco puso en marcha el programa Recrea, educación para refundar 20403. Dentro de esta amplia propuesta, se habían celebrado eventos en los que se fomentaba el uso de recursos tecnológicos -informáticos y mediáticos-, situación que se incrementó con la pandemia.

Desde un inicio, ambas estrategias dejaron fuera a los docentes que no estaban habilitados o que no tenían los recursos para conectarse a internet y seguir las capacita- ciones. En ese escenario, el abrupto cambio de la modalidad presencial a las modalidades no presenciales puso de nuevo sobre la agenda educativa un tema añejo: la falta de autonomía que los estudiantes tienen sobre el aprendizaje. Esto remite a la idea de que el interés por aprender no es motivado por las instituciones educativas y que, en estas, se continúa la reproducción de prácticas enfocadas en medir el aprendizaje por medio de trabajos o evidencias, y no tanto a través de procesos de generación de competencias y evaluación formativa. Paradójicamente, este fue un tema que el Gobierno federal se propuso revisar para el Consejo Técnico Escolar a mitad del confinamiento, cuando ya era tarde.

Este dilema sobre el interés de los alumnos por aprender $-\mathrm{o}$ no- resurge a la par de la pandemia, ya que el hecho de hacer las tareas en casa o, como se quiso manejar en ese momento, de continuar con el proceso educativo a lo lejos, pasó a ser un tópico que ahora involucra por primera vez la monitorización paralela de tutores y padres de familia. Otro tema en la agenda precontingencia fue que la mayoría de los docentes, más aún dentro del sistema de educación pública, no estaban acostumbrados a utilizar las aplicaciones educativas en el diseño e implementación de sus clases. Esta situación se vincula con el historial de programas tecnológicos fallidos que han transitado por la Secretaría de Educación Pública y con la falta de conti-

${ }_{3}^{3}$ Puede visitarse la página en http://edu.jalisco.gob.mx/consejo-tecnico-escolar/sites/edu.jalisco.gob.mx. consejo-tecnico-escolar/files/recrea agosto19 1.pdf. 
nuidad entre las gestiones administrativas (Arredondo Ramírez, 2020). El más cercano, el programa estatal México conectado, que se inició en el sexenio anterior -20122018-, dejó de proveer el servicio de internet gratuito desde principios de 2019 (Castañares, 2018). Si a ello se le suma la escasez de inversión en la renovación de equipos de cómputo e infraestructura informática, la consecuencia natural ha sido la falta de continuidad.

Por su parte, uno de los grandes retos al inicio de la contingencia estuvo en tratar de mantener en marcha una estrategia viable de vinculación con el proceso educativo regular y de contactar a las familias de los estudiantes. Justamente, a partir de que hace unos años se crearan los llamados Consejos de Participación Social, surgió la necesidad, por parte de las coordinaciones escolares, de sostener un contacto más estrecho con los padres de familia y tutores.

\section{¿Qué quedó tras las entrevistas?}

La percepción de los docentes dio lugar a dos grandes ejes en la codificación: aquellos a los que aquí llamamos de implicación personal y de implicación con los otros.

En el primero, los docentes se vieron como sujetos afectados individualmente por los problemas, los conflictos y las oportunidades del contexto, mientras que, en el segundo, como actores que vieron a los otros miembros de la comunidad educativa como sujetos de esas afectaciones -donde los otros están representados por sus estudiantes, los padres de familia, sus colegas y las autoridades educativas-. A su vez, en ambos ejes pudo observarse cómo se formaron, de manera simultánea y casi espontánea, tres grandes categorías de referencia perfectamente compartidas: el nivel logístico, el nivel tecnológico y el nivel contextual, niveles a partir de los cuales surgen las eventualidades posteriores.

El primer nivel, el logístico, tuvo como centro el recuento de experiencias relacionadas con la organización y el desempeño de labores, independientemente de las varias características de aquellas. El énfasis puesto por los docentes en esta categoría se sitúa en la resolución práctica de conflictos, en los costos implicados y en cómo fueron afrontando esas eventualidades.

Un ejemplo importante de este eje fue cómo estos docentes se vieron a sí mismos desde la idea del autodidactismo. Quienes tuvieron la oportunidad de migrar a las propuestas institucionales se contemplaron como autodidactas; desde su propia interpretación, el tránsito había implicado aprender a utilizar estas tecnologías no desde la oferta institucional, sino a partir de sus propios medios.

Esta percepción coincide parcialmente con el planteamiento de Sefo et al. (2017), quienes anotan que «ser autodidacta significa que el profesorado sea autónomo para actualizar su conocimiento digital» 
(p. 253), aunque, en este caso, los que lograron utilizar estas herramientas digitales por su cuenta lo hicieron más como una manera de salvar el escollo y cumplir con su trabajo que como un incentivo de crecimiento profesional.

Probablemente este hecho tenga que ver con la situación que presentan Barrón López et al. (2010), según la cual el autodidactismo entre los docentes mexicanos no ha sido una práctica común, ya que la labor de enseñar y educar se encuentra todavía muy arraigada en el método de enseñanza tradicional, en el que el docente es el único transmisor del conocimiento y se ubica tras la idea de que le basta con lo que tiene por conocimientos y medios de conocimiento.

Ya en el segundo eje, el tecnológico, la atención se concentró en una forma particular de esas eventualidades que, por su importancia en el proceso de migración, fueron separándose apriorísticamente de los problemas logísticos: el papel de las tecnologías y los ecosistemas tecnológicos en el surgimiento de conflictos, retos y oportunidades durante el proceso de transición.

En este sentido, los profesores refirieron que los tutoriales fueron su principal herramienta de referencia, mientras que los cursos ofrecidos por instancias gubernamentales y otros mecanismos de capacitación oficiales fueron rehuidos o soslayados sus conocimientos prácticos para interactuar con los medios de capacitación. Del mismo modo, este rechazo también ocurría porque, en la visión prospectiva de la crisis, sencillamente era más importante ocupar el poco tiempo disponible en resolver los apuros con las herramientas tecnológicas que se tenían o con las más rápidas de aprender.

En el tercer eje, el contextual, entraron en juego varias situaciones que tienen en común ser el resultado directo del migrar totalmente y comenzar a impartir docencia en esta nueva modalidad; se han considerado contextuales porque se trata de circunstancias periféricas pero derivadas del proceso de migración o directamente articuladas con él; son periféricas pues, aunque importantes en más de un sentido, no actúan como el centro o la bisagra de las narraciones. En esta categoría, entran las relaciones indirectas con los padres de familia, el atestiguamiento de las condiciones de vida de los estudiantes, el conocimiento del entorno institucional desde una perspectiva del ahora implicado o las sorpresas - para bien o para mal-detonadas por hechos circunstanciales en cualquier otro ámbito de la práctica docente.

\section{Principales hallazgos}

Las respuestas agrupadas por profesor $-\mathrm{y}$ sus respectivas materias- en torno a temas problemáticos, obtenidas a partir de las preguntas detonadoras, se explicitan en la siguiente tabla (Tabla 1): 
TALÍA CHÁVEZ PALENCIA Y RODRIGO GONZÁLEZ REYES / PERCEPCIONES SOBRE EL TRÁNSITO A MODALIDADES DE ENSEÑANZA NO

Tabla 1

Respuestas desagregadas de los profesores entrevistados

\begin{tabular}{|c|c|c|c|}
\hline $\begin{array}{c}\text { Docentes participantes } \\
\text { (Género / Materia que } \\
\text { imparte) }\end{array}$ & $\begin{array}{l}\text { Pregunta } 1 . \\
\text { Propuestas } \\
\text { gubernamentales }\end{array}$ & $\begin{array}{l}\text { Pregunta } 2 . \\
\text { Percepciones sobre la } \\
\text { modalidad }\end{array}$ & $\begin{array}{l}\text { Pregunta } 3 . \\
\text { Percepciones sobre la } \\
\text { enseñanza en pandemia }\end{array}$ \\
\hline $\begin{array}{l}\text { 1. Femenino/ } \\
\text { Ciencias }\end{array}$ & $\begin{array}{l}\text { La Secretaría de } \\
\text { Educación Jalisco se } \\
\text { tardó dos meses en } \\
\text { habilitar las cuentas } \\
\text { institucionales. Cargó } \\
\text { de manera errónea las } \\
\text { clases en Classroom. }\end{array}$ & Educación en línea & $\begin{array}{l}\text { Tuvo menos } \\
\text { participación de los } \\
\text { alumnos y fue difícil } \\
\text { contactar a varios. }\end{array}$ \\
\hline $\begin{array}{l}\text { 2. Femenino/ } \\
\text { Taller }\end{array}$ & $\begin{array}{l}\text { No sintió que estuviera } \\
\text { clara la propuesta. }\end{array}$ & Educación a distancia & $\begin{array}{l}\text { Al Classroom } \\
\text { únicamente entraron dos } \\
\text { de sus alumnos. }\end{array}$ \\
\hline $\begin{array}{l}\text { 3. Femenino / } \\
\text { Formación Cívica y Ética }\end{array}$ & $\begin{array}{l}\text { Las propuestas no están } \\
\text { aterrizadas al contexto } \\
\text { de las secundarias. }\end{array}$ & $\begin{array}{l}\text { «No estoy segura } \\
\text { de cómo se llama la } \\
\text { modalidad». }\end{array}$ & $\begin{array}{l}\text { Para ella ha sido muy } \\
\text { complicado aprender a } \\
\text { utilizar el Classroom y } \\
\text { el Meet, y le da miedo } \\
\text { que los alumnos se } \\
\text { den cuenta de sus } \\
\text { dificultades. }\end{array}$ \\
\hline $\begin{array}{l}\text { 4. Masculino / } \\
\text { Educación Física }\end{array}$ & $\begin{array}{l}\text { No ve Aprende en casa } \\
\text { como funcional por la } \\
\text { evaluación. El trabajo en } \\
\text { el Classroom le pareció } \\
\text { más funcional. }\end{array}$ & En línea & $\begin{array}{l}\text { Se ha organizado } \\
\text { para atender a todos } \\
\text { sus alumnos en el } \\
\text { Classroom, pero, en } \\
\text { realidad, no tiene } \\
\text { sesiones en directo. } \\
\text { Hay alumnos que no } \\
\text { comprenden bien } \\
\text { las instrucciones por } \\
\text { escrito. }\end{array}$ \\
\hline $\begin{array}{l}\text { 5. Masculino/ } \\
\text { Matemáticas }\end{array}$ & $\begin{array}{l}\text { Solo consideraron cómo } \\
\text { compartir la información } \\
\text { y que aprendan de los } \\
\text { contenidos. }\end{array}$ & $\begin{array}{l}\text { En línea y a distancia los } \\
\text { que no tienen acceso a } \\
\text { internet }\end{array}$ & $\begin{array}{l}\text { Funciona la educación en } \\
\text { línea, pero hace falta la } \\
\text { socialización entre pares. }\end{array}$ \\
\hline $\begin{array}{l}\text { 6. Masculino / } \\
\text { Formación Cívica y Ética }\end{array}$ & $\begin{array}{l}\text { Faltó seguimiento y } \\
\text { evaluación en estas } \\
\text { estrategias. }\end{array}$ & $\begin{array}{l}\text { En línea y a distancia los } \\
\text { que no tienen acceso a } \\
\text { internet }\end{array}$ & $\begin{array}{l}\text { Se tendría que garantizar } \\
\text { que todos los alumnos } \\
\text { tuvieran internet; si no, } \\
\text { es injusta la estrategia. }\end{array}$ \\
\hline
\end{tabular}




\begin{tabular}{|c|c|c|c|}
\hline $\begin{array}{c}\text { Docentes participantes } \\
\text { (Género / Materia que } \\
\text { imparte) }\end{array}$ & $\begin{array}{l}\text { Pregunta } 4 . \\
\text { Percepciones sobre la } \\
\text { comunidad educativa }\end{array}$ & $\begin{array}{c}\text { Pregunta } 5 . \\
\text { Aprendizaje sobre el } \\
\text { trabajo en linea }\end{array}$ & $\begin{array}{l}\text { Pregunta } 6 . \\
\text { Percepciones sobre la } \\
\text { seguridad }\end{array}$ \\
\hline $\begin{array}{l}\text { 1. Femenino/ } \\
\text { Ciencias }\end{array}$ & $\begin{array}{l}\text { Ante la falta de internet, } \\
\text { es a distancia, porque } \\
\text { tuvieron que imprimir los } \\
\text { trabajos. }\end{array}$ & $\begin{array}{l}\text { Incorporar nuevas } \\
\text { aplicaciones, como } \\
\text { TikTok. Ella lleva años } \\
\text { capacitándose en } \\
\text { herramientas en línea } \\
\text { porque trabaja en una } \\
\text { escuela privada. }\end{array}$ & $\begin{array}{l}\text { En un colegio, pusieron } \\
\text { un reglamento por los } \\
\text { riesgos de internet. Cree } \\
\text { que es un tema que se } \\
\text { tiene que regular en las } \\
\text { escuelas públicas. }\end{array}$ \\
\hline $\begin{array}{l}\text { 2. Femenino/ } \\
\text { Taller }\end{array}$ & $\begin{array}{l}\text { Los alumnos tienen } \\
\text { muchas carencias } \\
\text { económicas y los papás } \\
\text { solo aparecen para } \\
\text { reclamar calificaciones. }\end{array}$ & $\begin{array}{l}\text { Ella decidió usar el } \\
\text { Classroom porque es } \\
\text { lo que usan sus hijas } \\
\text { en la escuela privada } \\
\text { en la que estudian. El } \\
\text { Classroom ayuda a tener } \\
\text { las «evidencias» para } \\
\text { reclamaciones. }\end{array}$ & $\begin{array}{l}\text { Siente que hay } \\
\text { «íconos» que pueden } \\
\text { ser peligrosos, pero } \\
\text { no entiende cómo } \\
\text { funcionan. }\end{array}$ \\
\hline $\begin{array}{l}\text { 3. Femenino / } \\
\text { Formación Cívica y Ética }\end{array}$ & $\begin{array}{l}\text { Los alumnos están } \\
\text { confundidos; no } \\
\text { entienden bien cómo } \\
\text { funciona. }\end{array}$ & $\begin{array}{l}\text { Es complicado mantener } \\
\text { la motivación y el } \\
\text { contacto. }\end{array}$ & $\begin{array}{l}\text { «Esto es nuevo para mí; } \\
\text { no sé sobre los riesgos». }\end{array}$ \\
\hline $\begin{array}{l}\text { 4. Masculino / } \\
\text { Educación Fisica }\end{array}$ & $\begin{array}{l}\text { No todos sus alumnos } \\
\text { han aprendido a usar } \\
\text { el Classroom y ha sido } \\
\text { difíil contactar con } \\
\text { algunos. }\end{array}$ & $\begin{array}{l}\text { Siente que el Classroom } \\
\text { lo ha ayudado a } \\
\text { organizar su trabajo } \\
\text { y que calificar es más } \\
\text { sencillo. }\end{array}$ & $\begin{array}{l}\text { No cree que existan } \\
\text { riesgos. }\end{array}$ \\
\hline $\begin{array}{l}\text { 5. Masculino/ } \\
\text { Matemáticas }\end{array}$ & $\begin{array}{l}\text { Siente que los } \\
\text { videos para repetir } \\
\text { las instrucciones } \\
\text { favorecieron a los } \\
\text { alumnos. }\end{array}$ & $\begin{array}{l}\text { Aprendió de manera } \\
\text { autodidacta a utilizar el } \\
\text { Classroom y a hacer sus } \\
\text { videos en YouTube. }\end{array}$ & $\begin{array}{l}\text { No conoce tanto del } \\
\text { tema. }\end{array}$ \\
\hline $\begin{array}{l}\text { 6. Masculino / } \\
\text { Formación Cívica y Ética }\end{array}$ & $\begin{array}{l}\text { Tuvieron que enseñarles } \\
\text { a los alumnos desde } \\
\text { cómo enviar correos. } \\
\text { No tenían muchas } \\
\text { habilidades tecnológicas } \\
\text { educativas. }\end{array}$ & $\begin{array}{l}\text { Aprendió de manera } \\
\text { autodidacta y con ella de } \\
\text { sus compañeros. }\end{array}$ & $\begin{array}{l}\text { No conoce mucho del } \\
\text { tema, pero le preocupa } \\
\text { que no se habla de ello. }\end{array}$ \\
\hline
\end{tabular}


A partir de las respuestas de los entrevistados, descubrimos que las prioridades de las autoridades educativas y el profesorado no coincidieron en buena parte, lo que generó algunos problemas. En las entrevistas, uno de los encuentros más significativos fue descubrir que los motivos por los que decidieron involucrarse en las nuevas tecnologías no estaban relacionados con las presiones de la coordinación, sino con la preocupación personal por seguir atendiendo de la manera más óptima posible sus responsabilidades educativas. Desde ahí, varios docentes refirieron haber modificado su práctica como resultado de su experiencia en otros contextos; por ejemplo, una de ellas se involucró con Classroom a través de la experiencia indirecta de su hija, estudiante de secundaria, y sus propios cursos, mientras que otros comentaron que, en otras escuelas del sector privado en las que también laboran, fueron positivamente incentivados a utilizarlo, con lo cual aprendieron y exportaron su práctica a las escuelas públicas.

Con todo, aunque estos docentes interactuaron mucho con la plataforma Classroom, no existe una percepción generalizada ni transversal de que el cambio de modalidad implique, de manera natural, una mejora pedagógica. Lo que sí se manifestó, de modo consensuado, fue la percepción de que lograr la transición a modalidades no presenciales dio inicio a importantes prácticas autodidactas, aunque, tal como ya se analizó líneas atrás, esta experiencia estuvo más enfocada en la utilización pragmática de la tecnología que en los cambios sobre la práctica pedagógica y la superación a nivel personal y profesional.

Otro de los descubrimientos que se colocó sobre la mesa fue la importancia de los aprendizajes significativos y situados para los docentes implicados. Por ejemplo, en un incidente relatado por un docente de Matemáticas de tercer grado, este expuso que el rendimiento de sus estudiantes en esa materia fue sustancialmente más alto que en el resto de las asignaturas, a pesar de las carencias económicas y de acceso que ellos mismos habían manifestado.

En parte, él atribuye su «éxito» a una serie de videos que produjo en YouTube y a sus formularios de Google, pero también intuye que parte de la motivación de los alumnos se vinculó con la preocupación, muy genuina por parte de ellos, por prepararse bien ante la inminencia del examen de admisión a preparatoria, y que él, como docente, se diera cuenta de ello y lo aprovechara para adaptar sus contenidos a las condiciones tecnológicas que tenía a mano. Esta experiencia, desde su visión, reflejó la importancia de tomar en cuenta a los sujetos con los que se trabaja y sus contextos inmediatos, más allá de las meras condiciones técnicas. Así, el hecho de que los docentes consideren las inquietudes de los alumnos implica un compromiso por su parte y, por ende, un aprendizaje significativo. 
Por otro lado, algo también relevante que apareció en el curso de la investigación fue la manera en que los docentes pusieron en práctica la plataforma Classroom: de una forma casi nada coincidente con la propuesta oficial. Tal como fue señalado, la mayoría no utilizó esta plataforma como una manera de dar continuidad a sus clases en directo, sino como una oportunidad de tramitar sus cursos. Classroom fue visto por los entrevistados, ante todo, como una herramienta de gestión de los procesos de trabajo: sirvió para llevar el registro de las actividades, para almacenar datos y como medio de descarga eficiente; en otras palabras, representó un instrumento de gestión de «evidencias».

También, dentro del ejercicio de administración de trabajos, uno de los principales conflictos surgidos entre docentes y padres de familia fue la retroalimentación y calificación de las labores de los alumnos. El hecho de que los padres se involucraran más en los procesos educativos de sus hijos trajo como resultado una mayor presión para que docentes y autoridades explicitaran y transparentaran los procesos de evaluación.

Por otra parte, ahora desde la otra cara de la moneda, este acercamiento entre docentes y padres de familia tuvo también dos aspectos muy positivos: el trabajo docente fue revaluado y, para otros, también involucró la oportunidad de tener un primer acercamiento con muchos de los
La experiencia con las tecnologías educativas anteriormente planteadas abrió la puerta a la posibilidad, por parte de los docentes, de aprovechar estos medios, por ejemplo, para darle seguimiento a alumnos que, por situaciones médicas o contextuales, dejaron de ir a la escuela. Esto permite mantener una supervisión puntual y, a los alumnos, la posibilidad de continuar con sus estudios.

En un aspecto relativo a este último punto, algo que se buscó saber desde un principio en la investigación fue la concepción que estos docentes tenían sobre la idea de modalidades no presenciales, y esto fue lo que encontramos: los docentes asociaron la idea de educación a distancia a una modalidad no oficial que se pone en ejecución con alumnos expulsados por mala conducta. Esta estrategia, que no se encuentra escrita en los protocolos oficiales, está vigente y lleva practicándose varios años en distintas escuelas; al margen de esta aclaración hecha por ellos mismos sobre su modalidad a distancia, los maestros fueron incapaces de nominar el tipo de modalidad que están implementando.

Para terminar, consideramos que es importante regresar al eje de la dimensión tecnológica, pues en él se objetivan los hallazgos más relevantes de este estudio. Páginas atrás, se afirmó que el eje tecnológico se presentó como uno «abisagrado». Decimos esto porque el nivel tecnológico, como eje categorial descriptivo, operó haciendo las veces de gozne 
entre lo que afectó personalmente -en cualquier dimensión- a los docentes y lo que alteró su relación con los otros; por su parte, en un nivel inmediatamente más discreto, fue el lugar común en el que describieron la relación entre el nivel logístico y el contextual.

De otra manera, cuando un conflicto, un reto o una oportunidad apareció en los otros dos ejes - personal y contextual-, el lugar de resolución y regreso de sus narrativas fueron siempre las tecnologías; desde ahí, se puede afirmar que para estos docentes la experiencia integral de la migración siempre tuvo como centro la vuelta a la cuestión tecnológica, de tal suerte que ninguna explicación o descripción positiva o negativa sobre esta mudanza pudo quedar libre o por fuera de este eje.

Ahora bien, aunque esta recurrencia tácita a la cuestión tecnológica podría parecer de sentido común, estos docentes advirtieron que, en un inicio, la preocupación era más logística que tecnológica: les preocupaba más el cómo iban a organizar y desempeñar nuevas tareas en el plazo de lo inmediato que los medios en sí mismos.

Este hecho resulta entonces muy relevante -insistimos-, pues de alguna manera los docentes asumieron que estas tecnologías y entornos tecnológicos siempre han estado ahí, pero, sobre todo, que las habilidades técnicas necesarias para usarlos no eran mucho más complejas ni rebasaban los saberes que tenían hasta ese momento. Había que preocuparse por otras cosas.

Con todo, esta percepción fue rápidamente sustituida cuando una situación inesperada se volvió evidente e insoslayable: sus estudiantes, independientemente de género y grado, resultaron todo menos «nativos digitales». Aunque los docentes refieren que los alumnos presentan habilidades muy desarrolladas en el uso de redes sociales y un buen desenvolvimiento general en ciertos entornos digitales, una importante mayoría son prácticamente inoperantes en el manejo de ambientes y herramientas tales como el correo electrónico, las aulas virtuales o los sistemas de teleconferencia.

Con este fortuito descubrimiento, la atención dio un giro de 180 grados, y puso sobre el escenario la preocupación por los aspectos y las tecnologías que, ahora lo sabían, ya mediaban tanto los aspectos logísticos como los contextuales de su nueva realidad; y esto, de manera inquietante, se articulaba con otros problemas estructurales: la precariedad en el acceso material a infraestructuras tecnológicas necesarias para la continuidad del improvisado proyecto, tales como conexión a internet, disponibilidad de dispositivos electrónicos y funcionalidad de los equipos de cómputo (Arredondo Ramírez, 2020), por no hablar del analfabetismo tecnológico e informacional, también ya patente, de padres de familia y tutores. 


\section{Conclusión y reflexión}

Tal como se puede apreciar, a lo largo del estudio se hicieron evidentes situaciones y circunstancias que obviamente estarían ahí, como los problemas de acceso tecnológico o la precariedad en la formación tecnológica del profesorado. Pero, por otro lado, muchas de estas condiciones vinieron acompañadas de percepciones y estrategias de afrontamiento sorpresivas, que dan una idea del dinamismo y la plasticidad de una parte del profesorado.

$\mathrm{Al}$ momento en que se redactan estas conclusiones - noviembre de 2021- la pandemia sigue vigente y en desarrollo, y en los medios públicos las autoridades gubernamentales y la ciudadanía entablan distintos debates, casi todos controversiales, sobre las medidas por tomar ante el inicio del nuevo ciclo escolar. Lo único que sabemos es que gran parte de la forma en la que se irá o no solventando esta situación descansa en la capacidad de resiliencia y la manera de afrontar la adversidad que van asumiendo, en todo el mundo y no solo en México, los docentes.

Por su parte, la plataformización de la educación, en conjunto con el proceso general y transversal de la plataformización de la economía y la organización social en el orden de lo cotidiano (Srnicek, 2019), se imponen como el nuevo modelo de la sociedad de la información y como el impe- más básicos mecanismos de acceso a los sistemas globales de oportunidad.

En tanto, esperamos que esta instantánea que se tomó contribuya a ir tomando el pulso de la evolución de este problema y de las percepciones sobre este escenario, que, a ojos vistas, no será nada corto. 


\section{REFERENCIAS}

Acuerdo Número 02/03/20 [Secretaría de Educación Pública]. (2020, 16 de marzo). Por el que se suspenden las clases en las escuelas de educación preescolar, primaria, secundaria, normal y demás para la formación de maestros de educación básica del Sistema Educativo Nacional, así como aquellas de los tipos medio superior y superior dependientes de la Secretaría de Educación Pública. Diario Oficial de la Federación. https://www.dof. gob.mx/nota detalle.php?codigo $=5589479 \&$ fecha $=16 / 03 / 2020$

«Aprende en casa TV»: así funciona el programa educativo de la SEP durante la cuarentena por coronavirus. (2020, 23 de marzo). Infobae. https://www.infobae.com/ america/mexico/2020/03/23/aprende-en-casa-tv-asi-funciona-el-programa-educativo-de-la-sep-durante-la-cuarentena-por-coronavirus/

Arredondo Ramírez, P. (2020). La presencia de México Conectado en la educación básica. Comunicación y Sociedad, Artículo e7804. https://doi. org/10.32870/cys.v2020.7804

Auerbach, C. F. y Silverstein, L. B. (2003). Qualitative data: An introduction to coding and analysis. New York University Press.

Barrón López, J. V., Flores García, S., Ruiz Chávez, O. y Terrazas Porras, S. M. (2010). Autodidactismo: ¿una alternativa para una educación de calidad? Cultura Científica y Tecnológica, (41), 14-22. https://erevistas.uacj.mx/ojs/ index.php/culcyt/article/view/273
Castañares, I. (2018, 2 de marzo). 'México Conectado' queda blindado hasta 2019. El Financiero. https://www.elfinanciero.com.mx/empresas/mexico-conectado-queda-blindado-hasta-2019

Edwards, R. y Holland, J. (2013). What is qualitative interviewing? Bloomsbury Academic.

Gallagher, C. (2018). Telling it like it wasn't: The counterfactual imagination in history and fiction. The University of Chicago Press.

Gauntlett, D. (2007). Creative explorations: New approaches to identities and audiences. Routledge.

Hammersley, M. (1992). What's wrong with ethnography? Routledge.

Instituto Nacional de Estadística y Geografía. (2018). Delimitación de las zonas metropolitanas de México 2015. https://www.inegi.org.mx/contenidos/ productos/prod serv/contenidos/espanol/bvinegi/productos/nueva estruc/702825006792.pdf

Medina Mayagoitia, N., Navarro Casillas, A. M. y Flores Márquez, D. (2017). De la brecha a la inclusión digital y social: conceptos y discusiones. En A. I. Zermeño Flores (Coord.), Inclusión digital para la inclusión social. Contextos teóricos, modelos de intervención y experiencias de inclusión (pp. 43-62). Universidad de Colima.

Morgan, S. L. y Winship, C. (2014). Counterfactuals and causal inference: Methods and principles for social research (2. ${ }^{\text {a }}$ ed.). Cambridge University Press. (Trabajo original publicado en 2007) 
Saldaña, J. (2015). The coding manual for qualitative researchers ( $3 .^{\mathrm{a}}$ ed.). SAGE Publications. (Trabajo original publicado en 2009)

Schenone, M. (2020). La plataformización de la educación. Un esquema conceptual sobre la base de tres axiomas [Tesis de maestría, Universidad Torcuato Di Tella]. https://www.academia. edu/49306067/La plataformización de la educación un esquema conceptual sobre la base de 3 axiomas final

Sefo, K., Granados Romero, J. M., Lázaro, M.N. y Fernández-Larragueta, S. (2017). La formación del profesorado para un uso innovador de las TIC: un estudio de caso en la educación obligatoria en la provincia de Almería. Profesorado. Revista de Currículum y Formación del Profesorado, 21(4), 241-258. https://recyt.fecyt.es/index. $\mathrm{php} / \mathrm{profesorado/article/view/62503}$

Seidman, I. (2006). Interviewing as qualitative research: A guide for researchers in education and the social sciences $\left(3 .^{\mathrm{a}}\right.$ ed.). Teachers College Press.

Srnicek, N. (2019). Platform capitalism. Polity.

Watts, D. J. (2012). Everything is obvious: How common sense fails us. Currency. 